\title{
Urgencias oculares en niños y adultos: análisis epidemiológico de un centro latinoamericano
}

\section{Ocular emergencies in children and adults: epidemiological analysis of a Latin American center}

\author{
Alexander M. Martínez-Blanco* \\ Departamento de Oftalmología, Grupo VISOC, Universidad del Valle; Departamento de Oftalmología, Clínica Imbanaco Grupo Quirón Salud. Cali, \\ Colombia
}

\section{Resumen}

Objetivo: Analizar los principales diagnósticos oculares en niños y adultos que consultaron al departamento de urgencias en un centro de alta complejidad latinoamericano. Método: Análisis retrospectivo de pacientes que consultaron al departamento de urgencias por causas oculares en el Hospital Universitario del Valle Evaristo García E.S.E en Cali, Colombia, entre enero de 2015 y diciembre de 2020. Se analizaron la información demográfica y los diagnósticos de consulta de acuerdo con la Clasificación Internacional de Enfermedades 10. ${ }^{a}$ revisión. Resultados: Se atendieron 7938 visitas urgentes por condiciones oculares, de las cuales el $62.7 \%(n=4974)$ correspondieron a lesiones oculares traumáticas, $y$ entre las causas no traumáticas el $13.8 \%$ ( $n=1099)$ fueron por patologías del segmento anterior y el $9.5 \%$ ( $n=757)$ por patologías del segmento posterior. Los tres primeros diagnósticos de consulta fueron cuerpo extraño en la córnea o la conjuntiva ( $n=2697$, $34.0 \%)$, trauma contuso $(n=952,12.0 \%)$ y otras alteraciones de la córnea o de la conjuntiva $(n=755,9.5 \%)$. El $5.1 \%(n=137)$ de las consultas por cuerpo extraño correspondieron a eventos recurrentes. En pacientes menores de 18 años se encontró una mayor proporción de casos con traumas contusos, quemaduras y tumores oculares en comparación con otros grupos etarios ( $p$ < 0.05). En los mayores de 65 años se encontró un mayor porcentaje de visitas urgentes por hemorragia conjuntival, glaucoma secundario, degeneración macular y endoftalmitis $(p<0.05)$. Conclusiones: En niños, adolescentes y adultos, el trauma ocular fue el motivo de consulta más frecuente en el periodo de estudio, especialmente en los hombres. El cuerpo extraño en la córnea fue la causa más común. Las consultas por patologías del segmento anterior y posterior fueron más frecuentes en los mayores de 65 años.

Palabras clave: Urgencias oculares. Trauma ocular. Epidemiología. Cuerpo extraño en córnea. Quemaduras oculares. Glaucoma.

\section{Abstract}

Objective: To analyze eye diagnoses in children and adults admitted to an emergency room of a Latin American center. Method: Retrospective analysis of patients admitted to the emergency room of Hospital Universitario del Valle Evaristo García E.S.E in Cali, Colombia, from January 2015 through December 2020 due to eye conditions and disorders. Diagnoses were analyzed according to the International Classification of Diseases, tenth revision. Results: $A$ total of 7938 urgent visits due to eye diseases or disorders were analyzed. Of these $62.7 \%(n=4974)$ were blunt eye traumas, and among the non-traumatic

Correspondencia:

*Alexander M. Martínez-Blanco

Calle 5 36-08

Fecha de recepción: 21-06-2021

Fecha de aceptación: 08-10-2021

E-mail: alexander.m.martinez@ correounivalle.edu.co DOI: 10.24875/RMO.M21000207
Disponible en internet: 03-01-2022

Rev Mex Oftalmol. 2022;96(1):9-13

www.rmo.com.mx 0187-4519/@ 2021 Sociedad Mexicana de Oftalmología. Publicado por Permanyer. Este es un artículo open access bajo la licencia CC BY-NC-ND (http://creativecommons.org/licenses/by-nc-nd/4.0/). 
causes, $13.8 \%(n=1099)$ were anterior segment injuries, and $9.5 \%(n=757)$ were posterior segment injuries. The leading diagnoses were cornea/conjunctiva foreign body $(n=2697,34.0 \%)$, followed by eye contusion $(n=952,12.0 \%)$ and other disorders of the cornea/conjunctiva $(n=755,9.5 \%)$. Among the foreign body visits, $5.1 \%(n=137)$ were recurrent events. In patients under 18 years of age, more cases of contusions, eye burns, and tumors were reported compared to other age groups $(P<.05)$. In patients over 65 years of age, more urgent visits due to conjunctival hemorrhage, secondary glaucoma, macular degeneration, and endophthalmitis were reported $(P<.05)$. Conclusions: In children, adolescents, and adults, eye trauma was the most common cause for consultation in the study period, especially among men. The corneal foreign body was the most common mechanism. Visits due to anterior and posterior segment injuries were common in patients over 65 years.

Keywords: Eye emergencies. Eye trauma. Epidemiology. Cornea foreign body. Eye burns. Glaucoma.

\section{Introducción}

La discapacidad visual desempeña un papel importante en la calidad de vida de las personas, con costos directos e indirectos que repercuten en la sociedad'. Aunque la mayoría de las patologías oftalmológicas son tratadas de forma ambulatoria, existen síntomas y condiciones oculares que obligan al paciente a solicitar una consulta de urgencia, y que representan el $1-7.6 \%$ de todas las consultas ${ }^{2-4}$. La presencia de ojo rojo, la pérdida repentina de la agudeza visual y el trauma ocular son las causas oftalmológicas urgentes con más frecuencia reportadas ${ }^{5}$. Sin embargo, estas pueden variar de acuerdo con el contexto social y el sistema de salud de cada región.

La epidemiología de las consultas oftalmológicas de urgencia se ha sido bien descrita principalmente en países desarrollados ${ }^{6-8}$, lo que ha permitido realizar una mayor planeación y una mejor utilización de los recursos disponibles, así como preparar de forma eficiente a los servicios de urgencias para ofrecer una atención oportuna y el mejor tratamiento disponible, impactando positivamente en el pronóstico visual del paciente ${ }^{9}$.

En Latinoamérica, en especial en Colombia, el acceso a los servicios de salud especializados ambulatorios es restringido y los tiempos de oportunidad de atención pueden ser prolongados, lo que obliga a que una proporción de los casos con condiciones que pueden ser tratadas de forma ambulatoria busquen atención en las unidades de urgencias. Además, en otros contextos se ha descrito que el $40-70 \%$ de los pacientes consultan por condiciones oculares no urgentes $^{6,10-12}$, lo que congestiona la capacidad de respuesta de los equipos en las salas de urgencias. En consecuencia, un conocimiento profundo de la epidemiología de las condiciones oculares que requieren atención urgente puede ayudar a los tomadores de decisiones a generar políticas orientadas a mejorar la atención del paciente, así como a optimizar la utilización de los recursos en los sistemas de salud latinoamericanos. Por lo anterior, el objetivo de este estudio es analizar los principales diagnósticos oculares en niños y adultos que consultaron al departamento de urgencias en un centro latinoamericano de alta complejidad.

\section{Método}

Se realizó un análisis retrospectivo de todos los pacientes admitidos consecutivamente por condiciones oculares al departamento de urgencias del Hospital Universitario del Valle Evaristo García E.S.E en Cali, Colombia, del 1 de enero de 2015 al 31 de diciembre de 2020. Esta investigación se realizó en una institución hospitalaria pública de carácter universitario, que es un centro de referencia de atención médica para el suroccidente del país y atiende población con un nivel socioeconómico bajo y medio. El Comité de Ética de la Universidad del Valle aprobó la realización de este estudio (código 172-020), el cual fue conducido de acuerdo con los principios de la declaración de Helsinki.

Los casos se identificaron utilizando el sistema institucional de historias clínicas, filtrando aquellos diagnósticos de egreso relacionados con condiciones oculares utilizando la Clasificación Internacional de Enfermedades, 10. ${ }^{a}$ revisión (CIE-10). Los diagnósticos se agruparon en seis categorías: trauma, patologías del segmento anterior, patologías del segmento posterior, glaucoma, infecciones y otros.

Se realizó un análisis descriptivo utilizando el programa Stata versión 16.0 (StataCorp, College Station, TX, USA). Las variables cualitativas se resumieron utilizando frecuencias absolutas y porcentajes, y las variables cuantitativas con media y desviación estándar. La comparación de los diagnósticos de acuerdo con el sexo y el grupo etario se realizó con la prueba de ji al cuadrado o la prueba exacta de Fisher. Se consideró estadísticamente significativo un valor $p<0.05$. 


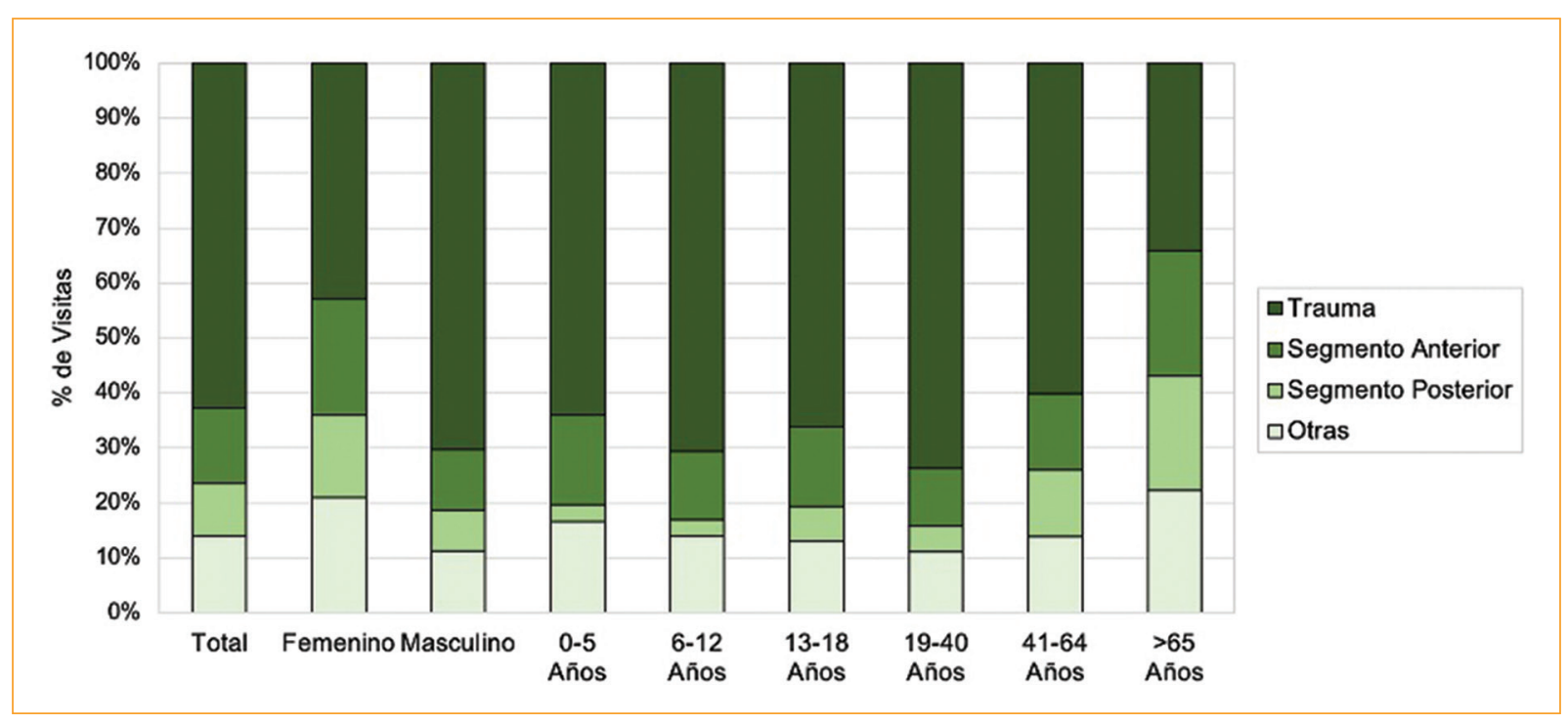

Figura 1. Distribución de los diagnósticos oftalmológicos de acuerdo con el sexo y la edad.

\section{Resultados}

Durante el periodo de estudio se atendieron 7938 visitas por condiciones oculares en el departamento de urgencias, con un número promedio de 1323 consultas por año. Entre todas las consultas urgentes, las condiciones oculares representaron un $2.6 \%(7938 / 303,002)$. La edad promedio de consulta fue de $41.4 \pm 19.8$ años y el $71.9 \%(n=5705)$ eran hombres. El motivo de consulta más frecuente fue el trauma ocular $(n=4974$, $62.7 \%$ ), seguido por condiciones del segmento anterior ( $n=1099,13.8 \%$ ), condiciones del segmento posterior $(n=757,9.5 \%)$ e infecciones $(n=332,4.1 \%)$. Las causas menos comunes fueron el glaucoma $(n=70,0.9 \%)$ y los tumores oculares $(n=46,0.6 \%)$. El $8.3 \%(660)$ de las visitas se debieron a otro tipo de afectaciones oculares diferentes de las descritas anteriormente. En la figura 1 se observa que el trauma ocular fue la condición más frecuente de consulta en hombres y mujeres, así como en todos los rangos de edad. Sin embargo, el porcentaje de trauma en los hombres fue 1.6 veces mayor que en las mujeres (masculino/femenino: $70.3 / 43.0 \%$ ), con una diferencia estadísticamente significativa $(p<0.001)$. El trauma ocular fue más frecuente en los rangos de edad de 6-12 años (70.6\%) y 19-40 años $(73.7 \%)$, disminuyendo en los mayores de 65 años (34.1\%). Las condiciones del segmento anterior y del segmento posterior fueron más comunes en las mujeres y en los adultos mayores de 65 años $(p<0.001)$.

Los cinco primeros diagnósticos específicos que llevaron a consultar a urgencias fueron cuerpo extraño en la córnea ( $n=2499,31.5 \%)$, trauma contuso ( $n=952$,
12.0\%), otras alteraciones de la córnea o de la conjuntiva ( $n=755,9.5 \%)$, trauma de párpados $(n=593,7.5 \%)$ y quemaduras $(n=523,6.6 \%)$. En los pacientes menores de 18 años se encontró una mayor proporción de casos de quemadura ocular, trauma contuso y tumores oculares $(p<0.05)$ en comparación con otros grupos etarios. En los mayores de 65 años se encontró un mayor porcentaje de visitas urgentes por hemorragia conjuntival, glaucoma secundario, degeneración macular y endoftalmitis $(p<$ 0.05). Los desprendimientos de retina se observaron principalmente después de los 40 años (Tabla 1).

La presencia de cuerpo extraño en el ojo se observó con mayor frecuencia entre los 19 y los 64 años $(p<0.05)$, con un total de 2697 (34.0\%) visitas urgentes en 2560 individuos; de estas, el $88.2 \%$ (2258) fueron de hombres con una edad promedio de $40.6 \pm 15.8$ años. El $5.1 \%(n=137)$ de las consultas por cuerpo extraño correspondieron a eventos recurrentes.

\section{Discusión}

El principal hallazgo de este estudio es que la presencia de un cuerpo extraño en el ojo es el motivo de consulta más frecuente en un departamento de urgencias de un hospital de alta complejidad en Cali, Colombia, representando una de cada tres visitas oftalmológicas. Al igual que en otros países, como Tailandia ${ }^{13}$, Taiwan ${ }^{14}$ y Pakistan ${ }^{2}$, las lesiones traumáticas en el ojo fueron la primera razón de consulta, contrario a lo reportado por países como los Estados Unidos de América ${ }^{6}$, España ${ }^{5,12}$ y Brasil ${ }^{15}$, donde las patologías infecciosas (p. ej., conjuntivitis) son el principal motivo. 
Tabla 1. Diagnósticos oftalmológicos específicos por edad

\begin{tabular}{|c|c|c|c|c|c|c|c|c|}
\hline Diagnóstico & 0-5 años & 6-12 años & 13-18 años & 19-40 años & 41-64 años & > 65 años & $\mathbf{p}$ & Total \\
\hline $\begin{array}{l}\text { Trauma } \\
\text { Cuerpo extraño en } \\
\text { córnea }\end{array}$ & $41(12.6)$ & $20(7.2)$ & $67(20.0)$ & $1130(38.3)$ & $1081(35.6)$ & $160(15.7)$ & $<0.001$ & 2499 (31.5) \\
\hline $\begin{array}{l}\text { Cuerpo extraño en } \\
\text { conjuntiva }\end{array}$ & $3(0.9)$ & $8(2.9)$ & $9(2.7)$ & $94(3.2)$ & $69(2.3)$ & $15(1.5)$ & 0.013 & $198(2.5)$ \\
\hline Quemadura & $32(9.8)$ & $32(11.5)$ & $30(8.9)$ & $216(7.3)$ & $170(5.6)$ & $43(4.2)$ & $<0.001$ & $523(6.6)$ \\
\hline Trauma contuso & $101(31.1)$ & $113(40.5)$ & $90(26.7)$ & $357(12.1)$ & $224(7.4)$ & $67(6.6)$ & $<0.001$ & $952(12.0)$ \\
\hline Trauma de párpados & $25(7.7)$ & $18(6.4)$ & $23(6.8)$ & $277(9.4)$ & $205(6.8)$ & $45(4.4)$ & $<0.001$ & $593(7.5)$ \\
\hline Trauma penetrante & $6(1.8)$ & $6(2.1)$ & $4(1.2)$ & $99(3.4)$ & $76(2.5)$ & $18(1.8)$ & 0.023 & $209(2.6)$ \\
\hline $\begin{array}{l}\text { Segmento anterior } \\
\text { Hemorragia } \\
\text { conjuntival }\end{array}$ & $12(3.7)$ & $8(2.9)$ & $9(2.7)$ & $61(2.1)$ & $147(4.8)$ & 107 (10.5) & $<0.001$ & $344(4.3)$ \\
\hline $\begin{array}{l}\text { Otras alteraciones } \\
\text { córnea/conjuntiva }\end{array}$ & $41(12.6)$ & $27(9.7)$ & $40(11.9)$ & $247(8.4)$ & $273(9.0)$ & $127(12.4)$ & $<0.001$ & 755 (9.5) \\
\hline $\begin{array}{l}\text { Segmento posterior } \\
\text { Desprendimiento } \\
\text { retina }\end{array}$ & $5(1.5)$ & $4(1.4)$ & $9(2.7)$ & $49(1.7)$ & $168(5.5)$ & $60(5.9)$ & $<0.001$ & $295(3.7)$ \\
\hline $\begin{array}{l}\text { Degeneración } \\
\text { macular }\end{array}$ & $0(0.0)$ & $0(0.0)$ & $0(0.0)$ & $0(0.0)$ & $15(0.5)$ & $58(5.7)$ & $<0.001$ & $77(0.9)$ \\
\hline Desgarro retina & $1(0.3)$ & $0(0.0)$ & $2(0.6)$ & $7(0.2)$ & $23(0.8)$ & $5(0.5)$ & 0.066 & $38(0.5)$ \\
\hline $\begin{array}{l}\text { Otros trastornos } \\
\text { retina/cuerpo vítreo }\end{array}$ & $4(1.2)$ & $4(1.4)$ & $10(3.0)$ & $80(2.7)$ & $163(5.4)$ & $90(8.8)$ & $<0.001$ & $351(4.4)$ \\
\hline \multicolumn{9}{|l|}{ Infecciones } \\
\hline Endoftalmitis & $7(2.1)$ & $5(1.8)$ & $1(0.3)$ & $27(0.9)$ & $45(1.5)$ & $42(4.1)$ & $<0.001$ & $127(1.6)$ \\
\hline Otras & $7(2.1)$ & $10(3.6)$ & $12(3.6)$ & $69(2.3)$ & $81(2.7)$ & $26(2.5)$ & 0.639 & $205(2.6)$ \\
\hline \multicolumn{9}{|l|}{ Glaucoma } \\
\hline Glaucoma secundario & $0(0.0)$ & $0(0.0)$ & $2(0.6)$ & $2(0.1)$ & $17(0.6)$ & $25(2.4)$ & $<0.001$ & $46(0.6)$ \\
\hline Cierre angular & $2(0.6)$ & $0(0.0)$ & $0(0.0)$ & $5(0.2)$ & $12(0.4)$ & $5(0.5)$ & 0.231 & $24(0.3)$ \\
\hline $\begin{array}{l}\text { Tumores } \\
\text { Tumores oculares }\end{array}$ & $16(4.9)$ & $1(0.4)$ & $1(0.3)$ & $8(0.3)$ & $9(0.3)$ & $11(1.1)$ & $<0.001$ & $46(0.6)$ \\
\hline \multicolumn{9}{|l|}{ Otros diagnósticos } \\
\hline Alteraciones visuales & $5(1.5)$ & $6(2.1)$ & $11(3.3)$ & 102 (3.5) & $123(4.1)$ & $58(5.7)$ & 0.003 & 305 (3.8) \\
\hline $\begin{array}{l}\text { Alteraciones } \\
\text { párpado/órbita }\end{array}$ & $2(0.6)$ & $5(1.8)$ & $3(0.9)$ & $30(1.0)$ & $37(1.2)$ & $7(0.8)$ & 0.530 & $84(1.1)$ \\
\hline $\begin{array}{l}\text { Otros trastornos no } \\
\text { especificados del ojo }\end{array}$ & $15(4.6)$ & $12(4.3)$ & $14(4.1)$ & $83(2.8)$ & $94(3.1)$ & $51(5.0)$ & 0.013 & $269(3.4)$ \\
\hline
\end{tabular}

El trauma ocular es considerado una causa importante de discapacidad visual y ceguera prevenible en todo el mundo, con un mayor número de casos de ceguera en los países en vías de desarrollo (75/100,000 en comparación con 9/100,000 en los países desarrollados) $)^{16}$. Similar a lo reportado por otros autores ${ }^{5,6,17}$, se encontró una mayor proporción de casos con trauma ocular entre los hombres en la segunda y la tercera décadas de la vida. Este comportamiento se debe a que las lesiones traumáticas en el globo ocular y las estructuras anexas generalmente se encuentran asociadas a actividades laborales.

El cuerpo extraño en la córnea o la conjuntiva fue el principal mecanismo de lesión ocular ${ }^{16}$. En este estudio se encontraron visitas recurrentes por esta causa, lo que sugiere que existe una carencia de programas orientados a educar al paciente en el uso de medidas de bioseguridad ocupacional con el fin de prevenir las recurrencias. La presencia de un cuerpo extraño puede tener consecuencias en la calidad visual de los pacientes, con un amplio espectro de afectaciones que incluyen problemas refractivos, queratitis y compromiso visual permanente, las cuales pueden variar según el tamaño de la lesión y el manejo realizado ${ }^{18}$.

Por otra parte, en niños y adolescentes se observó que la principal causa de consulta fue el trauma contuso, el cual se encuentra asociado con actividades de tipo recreativo ${ }^{19}$. Este hallazgo es diferente de lo reportado en España, donde la conjuntivitis fue el motivo de consulta más frecuente en este grupo etario, con un $29 \%$ de las visitas $^{20}$. En el mundo, en niños y adolescentes, la mayoría de las visitas urgentes son a causa de ojo rojo atribuible inicialmente a patologías infecciosas ${ }^{6}$. En nuestro contexto, este tipo de enfermedades son tratadas en la 
consulta externa, lo que explicaría la distribución de diagnósticos reportada en la presente investigación.

Otro hallazgo importante es la alta proporción de quemaduras oculares. En otro estudio realizado también en Cali, Colombia, se encontró que la mayoría de las quemaduras oculares eran por productos químicos $(62.7 \%)$, térmicos $(28.7 \%)$ y radiación ultravioleta $(6.8 \%)$, y que ocurrieron durante la realización de actividades en el hogar y en el trabajo, siendo los hombres los de mayor afectación, con una relación de 2:1 respecto a las mujeres ${ }^{21}$.

Nuestro estudio tiene ciertas limitaciones. Primero, debido a su carácter retrospectivo no se logró realizar una caracterización más precisa de los casos, como por ejemplo la sintomatología reportada, la ocupación del paciente o los mecanismos de la lesión, entre otros. Segundo, los datos presentados provienen de un solo centro latinoamericano ubicado en Cali, Colombia, por lo que la extrapolación de los resultados debe realizarse a la luz de cada contexto. $Y$ tercero, durante la revisión de los casos se identificó una alta clasificación de diagnósticos de egreso enmarcados en la categorización "Otros", por lo que se realiza un llamado a los oftalmólogos de urgencias a realizar una descripción más precisa de los casos haciendo uso de la CIE-10, lo que puede ser útil para futuras investigaciones.

\section{Conclusiones}

En niños, adolescentes y adultos, el trauma ocular fue el motivo de consulta más frecuente en el periodo de estudio, especialmente en los hombres. El cuerpo extraño en la córnea fue el mecanismo más común. Las consultas por patologías del segmento anterior y del segmento posterior fueron más comunes en los mayores de 65 años.

\section{Financiamiento}

El autor declara no recibir ningún tipo de financiación externa para realizar esta investigación.

\section{Conflicto de intereses}

El autor declara no tener conflictos de intereses.

\section{Responsabilidades éticas}

Protección de personas. El autor declara que los procedimientos seguidos se conformaron de acuerdo a las normas éticas del comité de experimentación humana responsable y de acuerdo con la Asociación Médica Mundial y la Declaración de Helsinki.

Confidencialidad de los datos. El autor declara que han seguido los protocolos de su centro de trabajo sobre la publicación de datos de pacientes.

Derecho a la privacidad y consentimiento informado. El autor obtuvo aprobación de los comités de Ética (código 172-020) y se ajusto a las exigencias exigidas en el manejo de la información.

\section{Bibliografía}

1. McKean-Cowdin R, Varma R, Wu J, Hays RD, Azen SP, Group LALES. Severity of visual field loss and health-related quality of life. Am J Ophthalmol. 2007;143:1013-23.

2. Baig R, Ahmad K, Zafar S, Khan NU, Ashfaq A. Frequency of ocular emergencies in a tertiary care setting in Karachi, Pakistan - It is time to reduce unnecessary visits. J Pak Med Assoc. 2018;68:1493-5.

3. Girard B, Bourcier F, Agdabede I, Laroche L. Activity and epidemiology in an ophthalmological emergency center. J Fr Ophtalmol. 2002;25:701-11.

4. Zafar S, Sebestyen K, Qureshi Z, Schein O, Johnson P, Srikumaran D, et al. National trends in imaging rates for eye-related emergency department visits in the United States. Am J Ophthalmol. 2020;211:114-22.

5. Galindo-Ferreiro A, Sánchez-Tocino H, Varela-Conde Y, Díez-Montero C, Belani-Raju M, García-Sanz R, et al. Ocular emergencies presenting to an emergency department in Central Spain from 2013 to 2018. Eur J Ophthalmol. 2021;31:748-53.

6. Channa R, Zafar SN, Canner JK, Haring RS, Schneider EB, Friedman DS. Epidemiology of eye-related emergency department visits. JAMA Ophthalmol. 2016;134:312-9.

7. Agrinier N, Conart J-B, Baudot A, Ameloot F, Angioi-Duprez K. Caractéristiques des patients examinés en urgence par un ophtalmologiste dans un $\mathrm{CHU}$ : résultats d'un étude transversale analytique identifiant des facteurs associés au caractère de véritable urgence. J Fr Ophtalmol. 2018;41:546-53.

8. McCarty CA, Fu CL, Taylor HR. Epidemiology of ocular trauma in Australia. Ophthalmology. 1999;106:1847-52.

9. Cheung CA, Rogers-Martel M, Golas L, Chepurny A, Martel JB, Martel JR Hospital-based ocular emergencies: epidemiology, treatment, and visual outcomes. Am J Emerg Med. 2014;32:221-4.

10. Carvalho $R$ de $S$, José NK. Ophthalmology emergency room at the University of São Paulo General Hospital: a tertiary hospital providing primary and secondary level care. Clinics (Sao Paulo). 2007;62:301-8.

11. Hau S, loannidis A, Masaoutis P, Verma S. Patterns of ophthalmological complaints presenting to a dedicated ophthalmic accident \& emergency department: inappropriate use and patients' perspective. Emerg Med J. 2008;25:740-4.

12. Domínguez-Serrano FB, Molina-Solana $P$, Infante-Cossío M, Sala-Turrens J, Seva-Silva N, Rodríguez-de-la-Rúa-Franch E. Ophthalmological emergencies. An epidemiological study: are resources been used properly? Arch Soc Esp Oftalmol. 2019;94:211-7.

13. Imsuwan I, Amnuaypattanapon K, Vongkittirux S, Imsuwan $Y$. The study of incidence and characteristics of patients with eye-related chief complaints at the emergency department of Thammasat University Hospital. Emerg Med Int. 2020;2020:4280543.

14. Hsu MH, Hsu CA, Hsiao SH, Chu D, Yen JC. Utilization of emergency ophthalmology services in Taiwan: a nationwide population study. Sci Rep. 2020;10:1-6.

15. Sen E, Celik S, Inanc M, Elgin U, Ozyurt B, Yılmazbas P. Seasonal distribution of ocular conditions treated at the emergency room: a 1-year prospective study. Arq Bras Oftalmol. 2018;81:116-9.

16. Feng K. Epidemiology of ocular trauma. En: Yan H, editor. Anatomy and examination in ocular trauma. Singapore: Springer; 2019. p. 105-22.

17. Fea A, Bosone A, Rolle T, Grignolo FM. Eye injuries in an Italian urban population: report of 10,620 cases admitted to an eye emergency department in Torino. Graefes Arch Clin Exp Ophthalmol. 2008;246:175-9.

18. Liu Y, Wang S, Li Y, Gong Q, Su G, Zhao J. Intraocular foreign bodies: clinical characteristics and prognostic factors influencing visual outcome and globe survival in 373 eyes. J Ophthalmol. 2019;2019:5208092.

19. Oiticica-Barbosa MM, Kasahara N. Eye trauma in children and adolescents: perspectives from a developing country and validation of the ocular trauma score. J Trop Pediatr. 2015;61:238-43.

20. Henriquez-Recine, Noval S, Zafra B, De Manuel S, Contreras I. Ocular emergencies in children: demographics, origin, symptoms, and most frequent diagnoses. J Ophthalmol. 2020;2020:6820454.

21. Ocampo HH, Contreras JC, Martínez A, Amaya CA, Bonilla-Escobar FJ. Quemaduras oculares en un centro de referencia oftalmológica de Santiago de Cali, Colombia. Colomb Med. 2008;39:210-8. 